\title{
Objets forts et rapports sociaux. Le cas des Yapere
} Minyanka

Power Objets and Social Relations : The Minyanka Yapèrè (Mali)

Jean-Paul Colleyn

\section{OpenEdition}

\section{Journals}

Édition électronique

URL : http://journals.openedition.org/span/1078

DOI : $10.4000 /$ span. 1078

ISSN : 2268-1558

Éditeur

École pratique des hautes études. Sciences humaines

\section{Édition imprimée}

Date de publication : 1 septembre 1987

Pagination : 221-262.

ISSN : 0294-7080

\section{Référence électronique}

Jean-Paul Colleyn, « Objets forts et rapports sociaux. Le cas des Yapere Minyanka », Systèmes de pensée en Afrique noire [En ligne], 8| 1987, mis en ligne le 07 octobre 2013, consulté le 01 mai 2019. URL : http://journals.openedition.org/span/1078; DOI : 10.4000/span.1078 


\title{
OBJETS FORTS ET RAPPORTS SOCIAUX
}

\author{
LE CAS DES YAPERE MINYANKA
}

par

Jean-Paul Colleyn

La fabrication d'objets rituels occupe dans la vie religieuse des Minyanka une place si importante que l'ethnographe ne peut éviter I'épineuse question des "fétiches" et du "feétichisme". Ces mots ont été tellement galvaudés que la plupart des anthropologues actuels évitent de les utiliser. Mais dans bien des séminaires d'anthropologie, ils viennent spontanément aux lèvres, accompagnés d'un petit signe figurant. les guillemets pour signaler que l'orateur n'est pas dupe de leurs connotations encombrantes. L'analyse de ces connotations a déjà été menée de main de mâtre par J.B. Pontalis et J. Pouillon ${ }^{1}$, mais il est utile d'y revenir. Une remontée dans le temps permet, en effet, d'expliquer à la fois la réticence de nos contemporains et l'étrange fascination qu'exerce sur eux cette notion dont ils se débarrassent difficilement. D'autre part, l'étude des matériaux de terrain minyanka à la lumière de certaines idées des théoriciens du "fétichisme" permettra peut-être de suggérer que les avatars de la notion de fétiche ne comportent pas que des aspects négatifs.

10bjets du fétichisme, Nouvelle Revue de Psychanalyse, $n^{\circ} 2,1970$, "Présentation", p.5-15 et "Fétiches sans fétichisme", p.135-147. 


\section{Préliminaires}

Voyons d'abord pourquoi les anthropologues d'aujourd'hui se défient du terme fétiche. Cette attitude ne date pas d'hier. Déjà en 1906, Alfred Haddon, Professeur à Cambridge, écrivait:

"The word fetishism has been so misused of late that ethnologists are apt to view it askance and hesitate to employ it in religious classifications. It has been stretched to such an extent in various directions that it has lost the definition and precision necessary for a scientific term" (A.Haddon, $1906: 64$ ).

"Le mot fétichisme a dernièrement été utilisé de façon si abusive que les ethnologues tendent à le considérer avec méfiance et hésitent à l'utiliser dans les classifications religieuses. Le sens en a été à tel point élargi qu'il n'a plus la définition et la précision nécessaire à un terme scientifique."

A la même époque, Marcel Mauss, critiquant les ouvrages de M. Dennett et $W$. Wundt, conseillait d'abandonner la notion, qui selon lui ne correspondait à rien de défini et devait disparâtre de la science:

"Quand on écrira l'histoire de la science et de l'ethnographie, on sera étonné du rôle indû et fortuit qu'une notion du genre de celle de fétiche a joué dans les travaux théoriques et descriptifs. Elle ne correspond qu'à un immense malentendu entre deux civilisations, l'africaine et l'européenne ; elle n'a d'autre fondement qu'une aveugle obéissance à l'usage colonial, aux langues franques parlées par les Européens sur la côte occidentale" (1907/1969: II, $244-246$

Le remède proposé par Mauss eût peut-être été pire que le mal. Il recommandait en effet de remplacer fétiche par mana. Tout en reconnaissant l'immensité de la dette de l'anthropologie moderne envers Mauss, Claude Levi-Strauss a bien montré que le recours à des notions de type "mana" consiste à expliquer l'inexplicable par l'inexpliqué (Levi-Strauss, 1950: XXXVIII-L). Quoi qu'il en soit, quatre-vingts ans plus tard, le terme fétiche est toujours vivant et la confusion s'est encore accrue. Après sa sévère mise en garde, Marcel Mauss lui-même avait continué à l'employer par commodité (Oeuvres, 1968: $84-85,365,375$, et 1969: 182, 184, 440). Les anthropologues voudraient bien changer de vocabulaire, mais aucun d'entre eux ne propose une conceptualisation capable de rendre compte des pratiques naguère "couvertes" par la notion de fétichisme. 
Bien qu'elle soit difficile à remplacer, cette terminologie semble irrémédiablement périmée en raison de l'ethnocentrisme des auteurs qui en ont assuré le succès. Fétiche vient du portugais feitiço et signifie originellement "chose fabriquée". Utilisé par les marchands du 15ème siècle pour désigner les charmes magiques et les amulettes africaines, il est passé dans le français colonial. C'est en 1760 que le Président de Brosses, dans son livre intitulé "Du culte des dieux fétiches", l'introduisit dans la toute jeune littérature ethnographique. Pour lui, il s'agissait d'une forme première de religion qui se caractérise par l'adoration directe d'objets. Comme l'a fait remarquer Jean Pouillon, de Brosses souligne l'absence de médiation : "il n'y a rien derrière le fétiche et qui serait symbolisé par lui" (Pouillon, 1970: 139).

Auguste Comte apparaît comme le premier grand théoricien du fétichisme, mais la définition qu'il en donne ne présente plus aujourd'hui qu'un intérêt historique. Dans son Cours de Philosophie Positive publié en 1830 et 1842, il imagine "une première enfance intellectuelle de l'humanitél caractérisée par le fétichisme. Celui-ci consiste à attribuer des qualités humaines à des êtres non humains ou à des objets. Pour Comte, pendant l'âge "fétichique", les faits chimériques l'emportent sur les faits réels. "Sous le fétichisme, disait-il, l'esprit humain est dans un état qui produit une sorte d'hallucination permanente où les plus absurdes croyances peuvent altérer l'observation directe" (Comte, 1908: V-93). Trente ans plus tard, E.B.Tylor, Professeur à Oxford, apporte une contribution théorique plus intéressante. Il donne le nom d'animisme à l'attribution d'une âme ou d'un esprit aux animaux, aux plantes ou aux choses. Le fétichisme apparâtt alors comme une subdivision de I'animisme rendant compte des esprits qui ne sont pas inhérents aux êtres ou aux objets, mais qui s'y incorporent.

"Comte s'en était servi (du terme fétiche) pour désigner une théorie générale de la religion primitive d'après laquelle les objets extérieurs sont animés par une vie analogue à celle de l'homme. Il m'a semblé qu'il valait mieux employer le terme animisme pour désigner la doctrine des esprits en général, et restreindre la signification du terme fétichisme à la partie qui lui appartient tout particuliè- 
rement, c'est-à-dire de s'en servir pour désigner la doctrine des esprits attachés à ces objets, ou des esprits exerçant une influence par I'entremise de ces objets" (Tylor, 1870/1876: II-187).

On notera, par rapport à de Brosses, que la distinction contenant/contenu prend le pas sur l'adoration directe d'objets. Une autre originalité de Tylor consiste à expliquer par la même théorie, celle de l'incarnation temporaire ou définitive des esprits et des âmes dans un corps solide, les phénomènes de possession et de fétichisme. Selon Tylor, le possédé n'est, à la limite, qu'une variante de l'incarnation fétichiste.

"D'une part, elle (la théorie de l'incarnation) permet d'expliquer les phénomènes d'exaltation et de dérangement morbide des facultés mentales (...). D'autre part, elle permet au sauvage de se débarrasser d'un esprit méchant en le logeant dans quelque corps étranger; elle lui permet aussi de porter avec lui dans un objet matériel un esprit qui lui rend des services; elle lui permet, pour adorer un esprit, de le faire entrer dans le corps d'un animal, dans' un bloc de bois, dans une image, dans un objet quelconque en un mot, qui contient l'esprit comme un vase contient un liquide et qu'il considère désormaîs comme sa divinité. Or, c'est là la véritable origine du fétichisme le plus strict" (id.:160).

Tylor et Comte s'accordent sur un point : ils distinguent les dieux des fétiches. Les seconds sont circonscrits à un objet particulier, alors que les premiers président à toutes sortes de phénomènes et s'incarnent, s'ils le désirent, dans différents corps ou objets. Plus précise que celle de Comte, la définition de Tylor demeure très large. Elle néglige le fait que le mot dérive de factiosus et désigne, à I'origine, un objet fabriqué. En délaissant le critère de la confection par la main de 1'homme, Tylor peut parler de "divinités fétiches" telles que le ciel, la lune et le soleil (id.:394).

Haddon (1906) s'attache lui aussi à distinguer la puissance de l'objet dans lequel il est incorporé. Continuant à parler de "low savages", de "backward peoples", de "unlightened mind", de "low grade of consciousness" et autres "child like capacity of make-believe", il a néanmoins le mérite d'étudier avec plus d'attention les objets en question. Comme Marcel Mauss, il note qu'il y a toujours une raison qui explique le choix de l'objet (rêve, songe, mythe), que celui-ci peut consister en un signe représentant une 
notion idéale (sans que la vraisemblance soit recherchée), qu'il est doté de pouvoirs mystérieux parce qu'il est le réceptacle d'une puissance. Haddon souligne aussi qu'on communique avec le fétiche, qu'on l'honore, qu'on le prie, qu'on Iui sacrifie, qu'on lui parle, qu'on le récompense, parce qu'il a une personnalité et une volonté. Cet aspect transactionnel de la relation était également souligné par Durkheim (1979: 52).

Le droit reconnu aux hommes de faire pression sur les entités religieuses et de tenter de les utiliser apparât comme une dimension majeure des religions africaines fondées sur la fabrication d'objets et le sacrifice. A la lecture des récits a'explorateurs, de marins, de marchands et de missionnaires, Hegel avait été heurté par l'inversion africaine du rapport de dépendance entre les dieux et les hommes. Le philosophe avait jugé la soumission des Africains à leurs dieux insuffisante pour qu'on puisse vraiment parler de religion (Hegel, 1830/1965: 254). Par la suite, Tylor enseigna aux anthropologues à évaluer de manière critique les récits des voyageurs, mais la distinction magie-religion servit encore longtemps un rationalisme ethnocentrique qui voulait voir dans les rites à finalité pratique de simples produits de l'ignorance. Pour l'ethnographe d'aujourd'hui, la question se pose autrement : comment une culture particulière envisage-t-elle les modalités de la circulation

d'informations et d'énergie entre les hommes et l'univers qui les entoure?

Le modèle des lois cosmiques ne saurait être seulement d'ordre intellectuel; il implique l'adhésion à un mode de vie et le recours à des pratiques jugées nécessaires en fonction d'un héritage. Les religions ouest-africaines ne sauraient se comprendre indépendamment d'une théorie implicite de la compétence sociale et de la mâ̂trise de I'événement. Les objets puissants dont elles recommandent I'usage ont toujours une finalité pratique. Ces objets créés par "les ancêtres" - une humanité supérieure, héroïque, thaumaturge, fondatricese donnent comme dignes d'être recherchés, servis et craints. Chargés des souhaits prononcés par les générations successives, recouverts du sang séché des sacrifices cycliques, ils résument toutes les 
recherches d'emprise et soulèvent la problématique du pouvoir. Se donner les moyens de manipuler les puissances qui y sont investies constitue un enjeu culturellement construit qui ne prend sens qu'au sein des relations sociales.

Chez les fondateurs de l'anthropologie, le fétichisme se maintenait dans les limites d'une conceptualisation propre à cette discipline. Peut-être le mot, expurgé de tout préjugé évolutionniste, aurait-il pu s'y maintenir et gagner en précision s'il n'avait connu par la suite un énorme succès dans la vulgate de la réflexion contemporaine. Comme le mot mythe, il s'est répandu dans les lieux communs d'une opinion confusément teintée de conscience critique par l'intégration dégradée des recherches en sciences humaines. Une expression à la mode, mise à toutes les sauces, résiste mal à la banalisation et aux dérives de sens. Aujourd'hui, dès qu'une cause est prise pour une autre dans quelque domaine que ce soit, on crie au fétichisme. Cet emploi vague et polémique de la notion permet de la trouver à la fois sous la plume de Pierre Hervé, pour dénoncer les dogmes du mouvement communiste (Hervé, 1955) et de Marshall Sahlins (Sahlins 1980: 116), pour critiquer certaines théories anthropologiques : "fétichisme de l'écologie", "fétichisme de la sociabilité"... Deux exemples parmi des milliers d'autres couvrant un champ immense. Sous l'influence du marxisme et de la psychanalyse, le "fétichisme" est devenu la formule passe-partout de la dénonciation des conformismes ineptes (cf. à ce sujet Baudrillard, 1971: 213).

Aujourd'hui comme hier, le mot dénote principalement l'erreur, surtout celle commise par d'autres, ailleurs ou dans le passé. Quand on l'emploie pour dénoncer les erreurs des adultes blancs, c'est seulement pour les montrer aussi flagrantes que celles des peuples "primitifs". Voilà donc les anthropologues soucieux d'objectivité dans la gêne ! Ils héritent d'un mot qui a servi à la sociologie évolutionniste pour définir un stade primitif de l'histoire des idées, au marxisme pour dénoncer les masques de la société marchande, et à la psychanalyse pour caractériser un type de perversion sexuelle. Comment désormais parler de fétichisme sans ressentir un certain malaise ? Pourtant le vocabulaire spécialisé ne propose 
aucun terme générique pour caractériser les religions qui recommandent le sacrifice sur des objets chargés de forces. En outre, alors que le mot est quasiment banni, son succès ancien continue à intriguer les chercheurs et à stimuler les réflexions. N'est-il pas remarquable qu'au tournant du siècle, les pratiques de sociétés identifiées comme "primitives" aient inspiré les théories chargées d'expliquer le comportement de l'homme moderne ? Le fait relève sans doute, selon la belle formule de Claude Lévi-Strauss, de ce "sentiment contradictoire de présence et d'étrangeté" que suscitent chez nous les usages exotiques (Lévi-Strauss, 1962: 277). Au moins dans une certaine mesure, les cultures sont intelligibles les unes aux autres même si on ne parle jamais tout à fait de la même chose. Si la métaphore fétichiste a fait une aussi belle carrière dans la sociologie de Marx et la psychanalyse, il vaut peut-être la peine qu'on s'y arrête. Le détournement de la notion de fétiche par la psychanalyse a permis un fécond retour d'idées. C'est en tout cas ce qu'enseignent les réflexions rassemblées, il y a seize ans déjà, par la Nouvelle Revue de Psychanalyse sous le titre espiègle d' "Objets du Fétichisme" (op. cité). Les contributions d'A. Adler et P. Bonnafé soulignent tout particulièrement la valeur de l'éclairage psychanalytique pour les anthropologues.

Mais en ce qui concerne la dimension sociale de la croyance magico-religieuse, une petite excursion du côté de Marx permet un renversement de perspective. Le retour en anthropologie de quelques idées proposées par Marx nous invite à renoncer à toute théorie du fétichisme - qu'il s'agisse d'une théorie exotique ou d'une théorie élaborée à partir de catégories occidentales - au profit d'une analyse des mécanismes de fétichisation. L'illustration de cette démarche par l'étude d'un cas particulier nous permettra de proposer une synthèse provisoire des informations relatives aux objets "forts" qui sont au coeur des pratiques rituelles des Minyanka du Mali. Danièle Jonckers, Philippe Jespers et moi-même menons depuis 1972 des recherches au sein de cette population établie dans la savane 
arborée du sud du pays ${ }^{2}$. Les Minyanka offrent l'exemple d'une société villageoise vivant de la culture du mil et du coton. De langue "voltaïque", ils sont cependant profondément influencés par les cultures mandé auxquelles ils ont emprunté de nombreuses institutions religieuses. Avec les Dogon, les Sénoufo et les Bobo, ils sont parmi ceux qui jusqu'ici ont le mieux résisté à l'impressionnante progression de l'Islam.

\section{La notion de yapèrè}

Chez les Minyanka, le dieu créateur est Klè (le Ciel). Bon et mauvais, donneur de vie et donneur de mort, associé à tout phénomène mais distant, il n'est pas un partenaire rituel privilégié. Comme dans beaucoup de religions ouest-africaines, il fait un peu figure de Dieu "g̀ la retraite". Il figure en tête de toutes les prières mais on ne lui demande qu'un lointain patronage pour fabriquer les objets appelés yapèrè 3 permettant d'agir sur des puissances plus manoeuvrables. Ces puissances peuvent être pensées indépendamment des objets, mais elles n'agissent qu'à l'issue d'un processus de fabrication : un yapèrè doit être "lié" et "mis" dans un village. Le dieu créateur, Klè, conçu comme un espace infini et enveloppant, peut se passer de support matériel, mais toutes les autres puissances s'appuient sur ces objets fabriqués. On ne se contente pas de les invoquer de manière abstraite : pour qu'elles se concrétisent localement, il faut fonder un sanctuaire, acquérir des yapèrè et les "nourrir" par des sacrifices. Les puissances elles-mêmes ne sont pas personnalisées et ne s'organisent pas en panthéon hiérarchisé. Essentiellement polymorphes, elles apparaissent simultanément, et de manière non contradictoire, comme une et multiples, mâles ou

2Des informations relatives à ces objets ont déjà été publiées dans Jespers 1976, 1979 et 1983 ; Jonckers 1976 et 1981 ; Colleyn et Jonckers 1983 ; Colleyn 1975 et 1982. Les précisions apportées par D. Jonckers (1986, sous presse) concernant les rapports masculinféminin et l'alliance complètent l'analyse proposée ici.

$3 \mathrm{pl}$. : yapèrlè ; par commodité, le mot ultérieurement sera laissé au singulier. 
femelles, immatérielles ou matérielles, bonnes ou mauvaisés. Elles tiennent du dieu puisqu'on leur demande la vie, la santé, la naissance d'une progéniture, la pluie, les récoltes, la prospérité, etc., mais aussi du monstre, de l'animal, de l'être humain et de l'objet magique. Quelques récits mythiques, les chants, les louanges et les prières les parent de qualités merveilleuses et les qualifient de fauve, d'antilope, de caïman, de femme rebelle, de chasseur, de sorcière, de mère, d'homme sincère, d'arbre, de vieille chose qui tue, de chose dangereuse, de singe mâle, de queue, d'organes sexuels, de lait nourricier, etc. Les puissances qui "prennent en bouche" en s'incarnant dans un possédé (Nya, Nankon) ou dans un porteur de masque habilité à parler en leur nom (Komo) présentent le plus de traits anthropomorphes. Elles discutent, négocient, se fâchent, manifestent toutes les humeurs propres à I'être humain.

Le même mot, yapèrè, dont aucune étymologie n'est établie, désigne aussi bien de petit objets personnels que les autels des grands cultes publics, les objets protecteurs que les objets maléfiques. D'un point de vue théorique il convient de distinguer ces deux catégories, bien que les recettes de fabrication se ressemblent et que les seconds puissent se développer à partir des premiers. Dans les deux cas, on stimule l'activité de l'objet en lui offrant un sacrifice et en prononçant une incantation magique, mais c'est seulement dans le cadre des grands cultes qu'on adresse une prière à une puissance nommée. Les noms Nya, Nankon, Komo, Manyan, Koro, Nama, Sandogno, Kono, Tyi-wara, Nantaganyo, Duga, Dagoro..., désignent les grands cultes, les puissances auxquelles ils se rapportent, les objets sur lesquels on sacrifie, et la société formée par les initiés.

Une foule de petites confréries relativement autonomes sont disséminées à travers le pays. Celles qui ont une origine commune entretiennent des rapports suivis et s'invitent mutuellement lors des grandes manifestations publiques. Tant que les prestations et les cadeaux dus à la société mère par la société fille n'ont pas été intégralement acquittés, celle-ci demeure sous tutelle, mais dès que ces obligations sont remplies, elle s'émancipe, fait à son tour "des filles" et finit par oublier son origine. Indépendamment 
de ces "généalogies", il arrive que des liens d'amitié, et même d'adoption, se nouent entre sociétés non apparentées. Les cérémonies peuvent mobiliser entre trois et dix sociétés provenant de villages voisins. Pour les initiés, une succession de fêtes tournantes animent la longue saison sèche, avec échanges de bière, de viande sacrificielle, de pagnes, d'argent et de noix de cola. Les sociétés qui sont liées se doivent aussi un secours mutuel au cas où, par exemple, un initié ne parviendrait pas à assurer la soudure entre deux récoltes. La réputation de certains sanctuaires ne dépasse pas la limite du quartier ; celle des plus réputés s'étend à tout le pays. A l'intérieur d'un même village, les sanctuaires de Nya, du Komo ou de toute autre puissance se livrent concurrence. Leur lutte d'influence marque la politique villageoise mais une règle d'ancienneté fondée sur l'ordre "d'entrée au village" fixe les préséances et limite les frictions.

Pour créer une société d'initiation dans un village, trois ou quatre chefs de famille se groupent, concluent un pacte d'amitié, et décident de fonder un sanctuaire. Cet accord, scellé par un serment (kali), intervient entre plusieurs personnes de bon renom, qui vont se répartir les fonctions et les charges. Le culte est toujours à la fois collectif (foroba) et privé (dyonkani) : il repose sur un large consensus, mais le matériel rituel n'appartient qu'à une seule famille. Celui qui a pris l'initiative de fonder un sanctuaire et a "payé" les autels en devient le détenteur. A ce titre, il est le chef de la société. De son vivant, il forme un ou deux héritiers et leur livre progressivement son savoir. La fonction s'hérite par rang d'âge, au sein du groupe de descendance en ligne paternelle. La règle de succession et d'héritage a résisté à la division des communautés familiales. Même si on ne cultive plus ensemble, sur le plan cérémoniel, l'unité du segment de lignage localisé se maintient. L'organisation sociale repose toujours sur l'aînesse et sur une valorisation de la séniorité.

Il serait impensable que la fonction de chef de culte se transmette verticalement, de père en fils, car le seul héritier légitime est l'ainé des descendants du fondateur. Le secret des autels appartient aux ancêtres, qui en restent les véritables mâtres et ne 
reconnaissent comme interlocuteur humain que l'ainé, celui qui leur est le plus proche4. Dans la pratique rituelle, comme dans la vie quotidienne, ce n'est pas le chef de culte qui orchestre les actions de tous. La vie institutionnelle se fonde sur une certaine séparation des pouvoirs entre chefs de culte, musiciens, bouffons, sacrificateurs, et, le cas échéant, possédés. Au sein de chaque classe d'initiés, une hiérarchie verticale, régie par l'aînesse, contrôle le comportement de chacun. L'idéal veut que ces classes recrutent dans des lignages différents, ce qui renforce la crédibilité de la société. Les différentes classes d'initiés entretiennent des "relations à plaisanteries" rituelles (senankuya) qui désamorcent les tensions.

Bien qu'il n'y ait pas de récits mythiques bien constitués, l'apparition des yapèrè dans l'histoire humaine résulte toujours d'un événement miraculeux : I'un aurait été dérobé à I'oryctérope, l'autre serait un don de Tyélèrè, la femme primordiale, un autre encore aurait été volé par un chasseur aux nains de la brousse (sighéshyinagbwèrè), etc. Bien sûr, ces objets ont une apparence dérisoire - leur aspect excrémentiel de boules noirâtres couvertes de sang séché fait même l'objet de plaisanteries - mais ils résonnent d'un écho prestigieux. Une des premières leçons prodiguées au nouvel initié enseigne de se méfier par dessus tout des choses "qui ne paient pas de mine" : "Ce qui se cache sous l'ongle peut tuer un homme". L'autel de Nya le plus redoutable n'est guère plus gros qu'un orteil. Les yapèrè "vivifient" (tshongo), ils donnent la force (baragha), la chance (karèsèghè), I'espoir (jigi). Mais pour garder leurs pouvoirs, ils doivent être nourris par le sang des sacrifices. La viande est toujours consommée par les hommes, mais le sacrifice se justifie exclusivement par 1'obligation de nourrir la puissance-objet qui absorbe la force contenue dans le sang. Ce don (kan) s'accompagne d'une prière prononcée par le sacrificateur au nom du sacrifiant.

4La primauté de l'âge n'admet qu'une seule nuance: quand au décès d'un chef lignager, le frère cadet (classificatoire) est plus jeune que le fils (classificatoire), une préséance formelle lui est accordée lors des sacrifices à caractère lignager. 
Les yapèrè sont bien davantage que des récipients (Tylor) ou des réceptacles (Haddon). Leur efficacité tient à leur procédure de fabrication. Bien que chacun d'entre eux ait sa spécialité, son travail (bara), ils tendent tous à se poser comme totalités et même à intégrer les autres puissances. Les grands cultes mobilisent de véritables formations de plusieurs dizaines de yapèrè, comme on parle de formation pour des avions ou des bateaux de guerre. Mais ce sont des choses que l'on cache, qu'on entoure de secrets, qu'on couvre d'enveloppes protectrices. En effet, leur puissance ne survit que si le détail de leur composition échappe aux investigations des adversaires de ceux qui les détiennent. Par conséquent, tout chef de famille cherche à renforcer son statut en se posant comme détenteur, exclusif ou non, d'un objet redoutable aux propriétés inconnues. Pour ce faire, il adopte deux procédures parallèles : une procédure d'alliance dans le cadre d'un ou plusieurs grands cultes et une procédure strjctement personnelle par l'acquisition de yapèrè propres.

Seule une très longue enquête pourrait révéler le yapèrè comme signe inséré dans une logique métonymique qui lui donne son sens. Le microcosme que forment les yapèrè participe d'un vaste système classificatoire qui intègre aussi la divination et les fabrications magiques. Déjà Mauss recommandait d'étudier l'objet qualifié de fétiche "tel qu'il est défini par le code de la magie et de la religion", mais ce mot d'ordre pré-structuraliste est particulièrement difficile à suivre. Comme dans les relations qu'on disait naguère totémiques, les oppositions fournies par les séries de yapèrè doivent bien former un système significatif, mais chaque individu $n^{\prime} e n$ détient qu'une partie. Nous savons qu'un système symbolique n'a nul besoin d'être totalement présent à la conscience pour fonctionner; mais davantage encore que pour la parenté ou la mythologie, la reconstitution du code qui intègre les modalités particulières, s'avère malaisée. Au fur et à mesure des progrès de l'investigation, le système semble s'éparpiller sous l'effet du secret et des spéculations liées aux stratégies sociales. Comme si un certain flou constituait une de ses lois de fonctionnement. A propos du sexe d'une puissance telle que Nya, de la composition de ses autels ou d'une 
recette médicinale, la caractéristique majeure semble être le polymorphisme et la polysémie. Dans ces conditions, il serait hâtif de considérer comme représentatives d'un culte, voire d'une culture toute entière, quelques modalités d'un ensemble certainement plus complexe. Pour prendre tout son sens, la notion structuraliste de transformation devrait ici intégrer une analyse sociologique du secret.

Bien qu'il s'agisse d'une "matière réservée", dont on ne parle pas librement, il est possible d'obtenir la liste des yapèrè de certains sanctuaires, leur composition et les incantations (klisi-kan) sans lesquelles leur fabrication serait vaine.

\section{Les autels de Nya}

Prenons l'exemple du culte de Nya qui est le plus répandu en pays minyanka. Le sanctuaire abrite trois sacs de coton renfermant plusieurs dizaines de yapèrè de la grosseur d'un poing. La plupart des initiés de plus de quarante ans connaissent "le nom et le travail" de chaque yapèrè mais au-delà, leurs connaissances varient fortement. Certains initiés sont respectés et craints à cause de I'étendue de leur savoir, d'autres passent pour ignorants. Personne ne reçoit jamais d'explications complètes et systématiques. Le savoir implique une longue quête, récompensée par des résultats proportionnels aux efforts consentis. On n'oblige personne à se perfectionner mais en principe, "si tu restes dans les affaires de yapèrè, tu n'en finis pas de chercher".

"Nya, dit-on, est un petit monde et il ne cesse de s'étendre". Ses autels forment un microcosme; il opère une synthèse de tous les autres cultes. C'est ainsi qu'on trouve parmi les yapèrè de Nya des formes miniatures des autels Kan-shoo (jarre du village), Ka-shigé (bois sacré), Dasiri (génie du village), Nankon (autel de la société des chasseurs), Manya (autel de guerre et de chasse), Koro (société d'initiation masculine), Duba (autel d'origine bobo), Serkolo (forme de Nya mêle), Nantaganyon (autel de la société des bouffons), Sandogno (autel "hyène" favorisant l'agriculture), etc. 
A partir d'une base commune, chaque famille de Nya s'est constituée une série spécifique d'autels, que viennent encore modifier les démarches de chaque chef de Nya. "Si tu t'y connais, dit-on, tu transformes Nya à ta main". Tout chef de Nya "court" : il travaille à renforcer ses autels par l'adjonction d'objets supplémentaires que l'on appelle yafaragha, "chose a joutée". Les frontières ethniques importent peu. Au cours de ses pérégrinations, un chef de Nya minyanka peut très bien acquérir un autel bambara en pays sénoufo et l'ajouter à son Nya. Pour ce faire, il doit sacrifier un poulet sur les sacs de Nya en disant : "J'ai cherché quelque chose à ajouter ; est-ce que cela peut être mis en toi ?" Si le poulet tombe sur le dos, le sacrifice est accepté et l'autel est incorporé à la batterie du culte. Celle-ci n'est donc fixe ni en ce qui concerne le contenu, ni en ce qui concerne le nombre. Voici cependant quelques observations générales, basées sur un inventaire des séries d'objets provenant de trois sanctuaires de Nya.

Les autels sont fabriqués par l'agglomération de fragments divers. A chaque étape de la confection, ces fragments sont liés par des fils rouges, noirs et blancs, et collés par différentes sortes de terre et de substances sacrificielles. Le sacrifice sanglant au-dessus de cet amalgame se fonde sur un système de rapprochements analogiques et sur une théorie de I'énergie. Le support de l'autel renferme en effet une énergie statique, tandis qu'une énergie fluide s'y ajoute sous forme d'eau, de bière, de crème de mil, de sang, de noix de cola et de piments mâchés, de plumes, de poils, de poudres végétales dont on enduit les objets ou avec lesquelles on les fume. Voyons d'abord les éléments constitutifs de ces autels, sachant qu'à chacun d'eux correspond une poudre médicinale conservée séparément. Administrée secrètement, cette préparation joue le rôle de vecteur, réel ou imaginaire, de l'efficacité de l'autel.

La plupart des autels ont pour base des racines : "les yapèrè, dit-on, ce sont des arbres". D'une manière générale, la racine est considérée comme un objet miraculeux qui crée la vie à partir du monde souterrain de la mort. Ces composants végétaux sont très secrets, non seulement en ce qui concerne les racines, mais aussi les feuilles, les écorces, le gui de différents arbres et la manière 
de les réduire en poudre. C'est en raison de la théorie de la force (nyama) que les diverses sortes de gui sont particulièrement prisées. Le gui pompe l'énergie pour l'accumuler tandis que le tronc qui le porte s'affaiblit. Vivant aux dépens d'une autre espèce, il ajoute les qualités de celle-ci aux siennes.

Tout yapèrè important comporte un noyau métallique (or, cuivre, ou fer). Beaucoup renferment des produits de la forge miniaturisés. La meilleure défense étant l'attaque, bien des yapèrè sont délibérément agressifs. Ces "noyaux" métalliques ont un aspect figuratif (arme ou outil) mais ils sont soustraits au regard par les racines et tous les produits dont on les entoure. Ces petites maquettes ultra-secrètes ne sont donc pas des signes destinés à un système de communication. Elles sont en quelque sorte des images-actes qui, intégrées dans une préparation magique, doivent conférer à celle-ci les qualités miraculeuses des premiers outils et armes révélés aux hommes par les esprits (jinè). Elles se présentent comme la projection d'un code sociologique fortement imprégné par le vocabulaire guerrier de l'époque précoloniale. On pourrait parler "d'armes symboliques", tant leurs noms et leur organisation empruntent à I'idéologie de la lance et du carquois. On trouve non seulement parmi les yapèrè de Nya, le "chef de guerre" (kashi-folo), avec ses "éclaireurs" (nyablabla), ses "lanciers" (tama-fè), ses "archers" (singbweghé-fè), et ses "fusiliers" (marfa-fè), mais aussi la "chaîne" (shoeh), la "Lance" (tama), le "sabre" (gnopara), la "hache" (katyèrèkè), le "couteau" (gmuu), la "flèche" (gmagha), les "pinces" (shèrèyè), le "casse-tête" (gbagha), la "bague" (kapelene), la "houe" (soo), et les "aiguilles" (museme). C'est notamment parce qu'il façonne ces objets métalliques que le forgeron bénéficie d'un prestige particulier. Héritier du premier homme apparu sur terre, il perpétue la lignée du héros civilisateur. Jadis, il se tenait à l'écart des champs de bataille. Il n'a aucune vocation au pouvoir, mais il mâtrise l'eau (il creusait les puits), les secrets de la terre (où il trouvait le minerai), le feu (essentiel au travail de la forge). De plus, comme fabricant des outils, des armes et des "noyaux" des autels, il est l'initiateur des techniques. 
L'enclume étant considérée comme une sorte de prototype de tous les yapèrè, le forgeron jouit d'une préséance qu'il tourne d'ailleurs généralement en dérision. Tout en passant pour le plus dangereux, l'autel tumpugno (l'enclume) a la particularité d'assurer les fonctions pacifiques de réconciliateur. C'est son pouvoir dissuasif qui l'habilite à imposer la médiation et à favoriser la concorde, à l'aide de deux autres autels appelés les "conciliateurs" (susutaghi). Un nombre important d'objets renferment des parcelles du corps des animaux sauvages dont ils portent les noms : calaos (tugbwagha et klèto), hyène (sandogno), vautour (tumpugno), oryctérope (timba). $\mathrm{Ce}$ bestiaire repose vraisemblablement sur un substrat mythologique dont ne subsiste qu'une imagerie éparse : l'oryctérope connaisseur de la terre souterraine, le petit calao appelant la pluie, le calao d'Abysinie célibataire malheureux, l'engoulevent ennemi des enfants, le lièvre rusé au souffle desséchant, la tourterelle bien informée, le cynocéphale ancêtre du forgeron, le vautour aîné des animaux, etc. Quelques récits appartenant à des genres littéraires divers évoquent encore ces figures, sans former toutefois de récit continu. Dans le procès de fabrication des yapèrè, l'intégration de parcelles particulièrement chargées de force prélevées sur le corps des animaux (têtes, queues, poils, pattes, plumes, griffes) constitue une opération essentielle. Les queues (nanga) forment une catégorie spéciale de yapèrè agressifs, qu'ils se présentent de manière autonome (comme objets personnels) ou qu'ils fassent partie de la panoplie du culte de Nya.

Parmi les autels de Nya, on reconnaît des petits casse-têtes de chasseur, modèles miniaturisés des autels que porte le possédé du Nankon, un autre culte important. Le casse-tête est l'archétype de l'arme avec laquelle le premier chasseur assenait le coup de grâce. Toute une série de yapèrè "décalquent" les techniques de chasse, avec leurs charmes, leurs pièges, leurs recettes magiques. Le chasseur est auréolé d'un prestige particulier. Il occupe une position de médiateur entre la brousse, conçue comme un lieu originel, source de toutes les connaissances, et le village. L'origine des yapèrè est presque toujours attribuée à un chasseur qui les aurait obtenus des animaux ou des génies (jinè) de la brousse. Connaisseur 
des plantes et des substances animales, le chasseur remplit souvent la fonction de guérisseur.

Quelques autels ont pour vocation d'assurer le succès du sanctuaire et d'attirer aux cérémonies un public nombreux. Ils sont fabriqués à base d'animaux et d'objets évoquant la foule (terre de fourmilière, fourmis, sauterelles) ou le pouvoir d'attirer des adeptes (figues, miel). Le principal autel porte le nom de kuyiri ("appelle le village"); comme son nom l'indique, il attire les villageois les jours de sacrifice. Il est fumé avec des plumes calcinées de vautour, matériau doublement propre à symboliser le grand nombre : les plumes sont aussi nombreuses sur le corps de I'oiseau que le sont les sauterelles convoitées par le vautour, lors des feux de brousse.

Plusieurs autels rattachent Nya à une chaîne d'ancêtres, qu'il s'agisse des ancêtres ayant habité le lieu ou des ancêtres liés à des sanctuaires plus anciens. Ils exigent le prélèvement de terre et de fragments divers, près de la plus ancienne porte du village, près du premiers puits, au carrefour, au cimetière, dans la plus ancienne cuisine, etc. On y incorpore, avec le temps, une phalange de l'index ou la rotule des ainés décédés. Le doigt symbolise leur enseignement qu'il faut aujourd'hui appliquer ; le genou symbolise les démarches qu'ils ont effectuées en brousse, à la recherche des "médicaments", ou dans d'autres villages, auprès de grands connaisseurs. Ces défunts ne figurent pas là en tant qu'ancêtres lignagers mais en tant qu'ancêtres fondateurs du culte. Ayant créé les yapère, ils gardent un certain contrôle sur eux. On peut même dire qu'ils y sont. intégrés. Dans les louanges, les puissances sont appelées par le nom de l'ancêtre fondateur : Koné-Diarra ou. Togola-Bankali pour Nya, Goïta et Koné pour le Nankon, Moriba pour le Komo, Menkoro Coulibali pour certains Tya-wara, etc. Le simple énoncé de ce nom et de celui d'anciens chefs de culte excite la puissance et stimule les objets actifs. Lors des processions semestrielles, les possédés de Nya se précipitent fréquemment vers le cimetière. Là, ils se couchent sur les tombes, écoutent les recommandations des aînés disparus et les transmettent aux vivants. 
L'autel Naghoo-nu, "mère des enfants", est responsable de l'appel des enfants sur terre. C'est lui qui fait de Nya le yiri-folo ("maître de l'appel") des enfants, qui reçoivent de ce fait un nom théophore, comme Nyazyé, Nyanyéré, Nyangolo, Warazyé, etc. Ce mécanisme assure à la fois la réincarnation des principes spirituels des ancêtres et la perpétuation du culte. Les enfants apparaissent à leurs propres yeux et à ceux de leur entourage comme consubstantiellement liés à la puissance qui les a appelés.

D'autres yapèrè protègent et favorisent les talents des différentes classes d'initiés, telles que les chanteurs, les bouffons et les possédés. La crise de possession ne saurait se concevoir sans l'intervention de certains objets. Les yapèrè-joro ("autels qui parlent") sont chargés de recruter les possédés, de les former et de leur dicter les messages de Nya. On dit qu'ils "donnent une bouche à Nya". Le tuntun-jyé (message-refuser), dont la poudre comporte des substances hallucinogènes, ramène l'individu "choisi par Nya" qui ferait mine de refuser le rôle de possédé à de meilleures dispositions.

Autre paradigme, celui de la sorcellerie qui démontre que si Nya peut lutter contre ce fléau, c'est en tant que sorcier suprême. Les Minyanka groupent sous le même terme, sinkama, deux types d'activités et de représentations dont la distinction witch/sorcerer ne suffit pas à rendre compte. Le premier donne forme à la crainte d'une persécution du groupe lignager par un ennemi intérieur clandestin (surtout féminin) capable de se livrer à des meurtres sacrificiels invisibles. Le second exprime la lutte d'influence magicoreligieuse à laquelle, dans une certaine mesure, tout le monde se livre. Il existe en effet toute une panoplie d'attaques occultes, dont la plupart s'appuient sur les yapère. Plusieurs autels typiques de la sinkama sont présents dans le matériel de Nya : nampogho, kortè, benjugu, satyè, sinkamashoo, etc. En énonçant le nom d'un ennemi et en prononçant une incantation spéciale, on peut "l'attacher" sur un yapèrè dans l'intention de le tuer ou de le rendre malade. Le yapèrè des uns peut done faire le malheur des autres : je peux être tué par le yapèrè de mon ennemi. Certains objets permettent 
d'attaquer des proches, d'autres des individus lointains. Leur nom dénote le degré de proximité de la cible : le cercle des amis (nyigbo, amis-tuer), la parenté (tshi-gbo, famille-tuer), les "étrangers" (kin-gbo, autres-tuer), les ennemis déclarés (jugu-gbo, ennemi-tuer), etc. Ce sont les yapèrè appelés shikorlo (chasseurs de brousse) qui permettent à Nya d'attaquer les sorciers.

Pour fabriquer les autels de Nya et d'autres puissances liées à la lutte anti-sorcellerie il fallait autrefois une double mort humaine, I'une perpétrée par les hommes et l'autre par la puissance elle-même. Le sacrifice d'un captif de guerre permettait la confection des autels qui exigent des poudres provenant d'organes humains pilés : coeur, foie, vésicule biliaire, os, etc. Longtemps après la pacification, ces poudres ont continué à circuler, car en raison d'une efficacité métonymique, de très petites quantités peuvent suffire. I'impossibilité de renouveler ces ingrédients compte aujourd'hui parmi les facteurs qui compromettent la confection des autels.

La seconde mort $n^{\prime}$ est pas considérée comme un sacrifice: elle était, dit-on, provoquée par la puissance, sans intervention humaine. Pendant que l'on fabrique en brousse, à proximité du village, les derniers autels, il faut qu'il meure quelqu'un "dont on connât le nom". Tant que ce décès n'a pas lieu, les autels ne peuvent entrer au village. En effet, leur finition exige un peu de terre provenant d'une tombe fraîchement creusée et le fil servant à lier les bois du brancard mortuaire. Ceux qui fabriquent les autels cachent ces derniers en brousse et attendent l'annonce d'un décès. Toute mort survenant pendant cette période est immanquablement attribuée à la puissance dont les autels sont en gestation. C'est ce qui vaut à ce Nya sa réputation de sérieux, en qualité de tueur et de sorcier suprême. En s'installant au village, le complexe puissance + autels s'impose par un acte comparable à ceux que son entrée a pour but de prévenir. Le rite d'installation serre au plus près l'acte défendu. Il révèle que la puissance n'est ni bonne ni mauvaise mais forte. Sa violence est propre à signifier l'arbitraire qui est l'essence même de tout pouvoir supérieur. 


\section{Echange de message et transfert d'énergie}

Pour être maintenus en vie, ces objets doivent être nourris par le sang de sacrifices au cours de grandes cérémonies semestrielles. Ces rites permettent d'entretenir la force statique que renferme le support de l'objet par l'adjonction d'une énergie fluide contenue dans des matières liquides. La notion de yapèrè en tant qu'objet fort ne peut se concevoir sans effusion de sang. Le sacrifice ne suffit pas pour créer un yapèrè, mais il n'est guère de yapèrè qui ne doive périodiquement être enduit de sang. Il faut "nourrir" les puissances religieuses pour les services qu'elles rendent, le premier de ces services étant d'épargner leurs adeptes et de maintenir en vie ceux qu'elles ont "appelés" sur terre. Chaque objet ayant ses interdits, il faut aussi "réparer" les offenses ; travail d'autant plus délicat que l'on n'est pas toujours informé des erreurs commises.

Le sacrifice et le traitement des autels postulent donc une circulation de messages : les hommes communiquent avec les puissances religieuses, généralement en demandant la bienveillance de Klé (Dieu) et en passant par l'intermédiaire des ancêtres. Par la prière, le message va des hommes vers la puissance : dans le sens inverse, il s'exprime par la ảivination et la possession. Le sacrifice lui-même admet une dimension divinatoire. Chaque fois que l'on offre un chien à Nya, il faut aussi sacrifier "le poulet de la tête du chien". C'est la position de ce poulet à l'agonie qui indique si le chien a été accepté, le signal fonctionnant sur le mode binaire : positif (le poulet tombe sur le dos) / négatif (il ne tombe pas sur le dos). Mais la communication implique aussi une circulation d'énergie. Le terme nyama, connu et compris dans une bonne partie de I'Afrique de l'Ouest, rend compte de cette conception de la force vitale.

En fonction des éléments entrant dans la composition des yapèrè et de l'adéquation des sacrifices, le nyama réalise les "travaux" spécialisés des différents autels. Le rite sacrificiel vise à le canaliser, à l'attirer, à le détourner, à le stocker. L'aspect énergétique est essentiel à la compréhension de la notion de yapèrè: celui-ci se charge de la force libérée par la mort violente et 
véhiculée par le sang. L'analyse de Marcel Griaule à propos des Dogon vaut certainement pour les Malinke, les Bamana et les Minyanka: "L'autel est le carrefour puissance-victime et, par suite, puissance-sacrifiant. Il est aussi le réservoir où boit la puissance, où puise le sacrifiant. Il représente de plus la puissance". (Griaule, 1940: 27-130).

C'est évidemment parce qu'il est une substance fluide contenue dans le corps des êtres vivants et dont toute "fuite" peut entrainer la mort, que le sang apparait comme support de la force vitale. Une force qui n'est jamais aussi visible et présente que lorsqu'elle est menacée de disparâttre. L'effusion de sang est précédée d'une offrande d'eau qui donne son nom et résume l'ensemble de la séquence sacrificielle. On dit par exemple : "le jour où on a versé l'eau à Nya", ou bien "Nya, voici ton eau, accepte-la de jour comme de nuit...". Dans une deuxième offrande, l'eau est associée au grain, sous forme de crème de mil (dègè) et de bière, elle-même définie comme de "l'eau de mil". Le symbolisme de ce geste est clair : l'aspersion de quelques gouttes suffit pour relancer le cycle cosmique. Mais si l'eau apparâ̂t comme l'élément premier (le plus ancien), elle est vouée à disparaître. Ne pouvant permettre le stockage de I'énergie, elle annonce le recours au sang, une substance plus pâteuse, qui peut être pétrie et collée sur un support solide. Lors du sacrifice à Nya, l'eau est versée par terre : "Nya, voici ton eau, je la verse à tes pieds, non sur la tête". Le sang, lui, est versé sur les yapèrè qui ont été déposés dans de grandes jarres. On les "crépit" (wologho) de sang, à la main et ce sang ne s'écoule pas complètement. Il "s'arrête sur le yapèrè" qui, à force, se recouvre d'une croûte noirâtre et prend du volume. Tout se passe comme si le meurtre sacrificiel visait à retenir, à fixer, à accumuler la force libérée par la "liquidation" d'êtres vivants. Par la mort, ceux-ci se déchargent d'une puissance qui ne peut se conserver qu'en des objets spécialement conçus pour l'absorber. La fabrication du yapèrè elle-même, puis son entretien, passe par un de ces actes violents dont la finalité n'est pas tant l'oblation (l'offre) que l'effort de rétention des forces dégagées par I'égorgement. 


\section{Aspects magiques de la pratique religieuse}

En dehors des cérémonies semestrielles, les chefs de Nya et les initiés importants peuvent avoir besoin d'un des autels de leur batterie de culte, pour un "travail" particulier. Ils procèdent alors, à l'intérieur du sanctuaire, à des sacrifices visant à une efficacité précise. Au lieu des poulets et des chiens du culte public, ils égorgent en secret, selon une procédure rigoureuse, des lézards, des margouillats, des caméléons, des souris, etc. Plutôt qu'un rite religieux, ces actes s'apparentent aux sortilèges, charmes, philtres et poisons. Dans ces opérations, le recours à des poudres, les incantations, l'énoncé du nom de la personne visée, l'adjonction d'éléments métonymiques "valant pour cette personne" (excréments, téguments, vêtements, traces, etc.), passent pour essentiels.

Le mot wèrè, employé dans le même contexte que yapèrè, évoque les recettes de fabrication des autels et des médicaments qui leur correspondent. Wèrè et yapèrè sont quasiment interchangeables, mais le premier signifie littéralement "feuilles", et par extension remède, médicament, autel, culte. Quand on dit "wèrè", les substances végétales liées à l'objet de culte viennent immédiatement à l'esprit. Les Minyanka francophones traduisent souvent le mot par remède. C'est une solution acceptable pour peu qu'on I'entende comme remède à tous les problèmes que pose la vie.

Shibé, qui signifie "poudres", correspond à la notion africaine de médicament. Il peut s'agir de drogues, de poison ou de substances plus magiques que réellement actives sur le plan pharmacologique. Ces poudres sont aussi bien destinées aux yapèrè qu'aux hommes. Les yapèrè recouverts de sang frais sont "renforcés" par le saupoudrage ou la fumigation de préparations dont la recette demeure rigoureusement secrète. Pour les chefs de culte, la connaissance de ces substances est si importante qu'un aphorisme dit : "Si tu entends qu'un yapèrè est bon, c'est grâce aux poudres à verser".

Le chef de Nya est à la fois savant, prêtre, guérisseur, devin et, d'une certaine manière, sorcier (sikan-folo). L'acquisition de yapèrè n'est pas un acte ponctuel : c'est une entreprise de stockage 
de forces et d'accumulation de puissance de longue haleine. Les pouvoirs sur l'invisible doivent faire leurs preuves dans le monde visible de la vie quotidienne : il faut que les adeptes du culte se sentent protégés. Quand le chef du culte acquiert les autels, il doit fournir un effort personnel pour les renforcer et connâtre les poudres végétales indispensables à leur efficacité. S'il ne connâtt pas les arbres correspondant à chaque catégorie d'autels et les recettes de fabrication, son sanctuaire est voué à péricliter. Quand sur une longue période les chefs de culte se révèlent compétents, les autels accumulent une puissance formidable, un peu comme si les efforts de tous ceux qui se sont succédés à cette chefferie avaient été capitalisés. Une sorte de valeur ajoutée 5 marque chaque transfert de matériel, chaque nouveau chef déployant toute son habilité à renforcer la crédibilité de ses autels. Aussi le chef de Nya affecte-t-il une part de ses ressources à la recherche de ce savoir supplémentaire, qu'on appelle shomalé. Littéralement, le mot signifie "ce qu'on achète pour mettre". Voici comment un chef de Nya explique ce que doit faire le nouveau chef de sanctuaire quand on lui donne les autels :

"Dès qu'on a fini de les nouer pour te les donner, toi-même, tu mets la vérité en toi et tu fais le travail afin que Nya ait une bonne renommée. Si tu les laisses là-bas en te bornant à sacrifier des chiens dessus sans chercher les arbres, à quoi Nya va-t-il te servir ? Ce n'est que de la viande que tu tues bêtement pour manger. Depuis que Nya a été attaché, toi chef de Nya, su tu n'es pas arrivé à connâ̂tre le shomalé, que connais-tu de Nya ? Tu ne connais rien de Nya". (Wolobougou, 1984).

En fonction du savoir-faire du chef de culte et de ceux qui l'assistent, les yapèrè acquièrent une sorte de cote; la concurrence est sévère. A la manière d'une entreprise dont les gestionnaires manifestent un talent variable, les sanctuaires connaissent des hauts et des bas au gré des ajouts judicieux ou des erreurs des hauts responsables. Ces derniers, en tant qu'individus forts accordant leur appui ̀̀ ceux qui se mettent sous leur protection, s'offrent

5L'expression m'a été suggérée par A. Zempléni, qui a bien voulu lire une première version de ce texte. 
évidemment comme cibles. Aussi les grands initiés disposent-ils, en plus des autels placés dans les sacs du sanctuaire et dont la somme porte le nom de Nya, d'objets visant à assurer leur sécurité et celle de leurs proches. Chacun garde, par exemple, dans un coin discret de sa case, un autel qui porte le joli nom de Nyusigengné, "cale-tête", parce qu'il "soutient" et permet de dormir en paix.

\section{La réification des rapports sociaux}

L'intérêt véritable de l'ethnologie consiste à déceler des logiques que l'on puisse comparer. Partir de ce que les Minyanka disent et de ce qu'ils se représentent s'imposait comme une étape nécessaire afin de dresser, comme dirait Mauss, le "catalogue des catégories" dont ils se servent. L'analyse doit ensuite dépasser le point de vue selon lequel le yapèrè semble tout-puissant et démonter l'opération qui produit cette surestimation. C'est là que les investigations de Karl Marx relatives à la fétichisation de la marchandise et de la monnaie ne manquent pas d'intérêt.

Il ne s'agit pas de défendre une démarche économiste qui privilégierait les forces productives et un aspect des rapports sociaux pour les ériger en facteur causal unique. Aujourd'hui, souligner la difficulté, voire l'impossibilité de séparer l'économie de la parenté et de la religion est devenu une banalité. Trop d'auteurs bien informés des travaux de Marx ont montré la nécessité de dépasser la théorie du symbolique considéré comme simple reflet des pratiques sociales pour qu'il vaille la peine d'y revenir. Dans la réflexion anthropologique contemporaine, il semble acquis que toute tentative d'opposer la pensée et la matière aboutit à une impasse. Ce qui nous intéresse chez Marx, c'est son effort pour comprendre comment un objet conventionnel paraît chargé de forces infuses. Les yapèrè sont inséparables des rapports sociaux, mais les multiples enveloppes, matérielles, sociales, et symboliques dont ils sont recouverts contribuent à les présenter comme des corps dotés de pouvoirs autonomes. Si, après avoir découvert la théorie de la valeur, Marx s'est servi de la métaphore du fétiche religieux pour caractériser la 
marchandise, c'est que dans un cas comme dans l'autre, les rapports sociaux impliquent la création d'un objet qui, bien qu'étant l'oeuvre des hommes, leur échappe et les domine.

"La forme valeur et le rapport de valeur des produits du travail n'ont absolument rien à faire avec leur nature physique. C'est seulement un rapport social déterminé des hommes entre eux qui revêt ici pour eux la forme fantastique d'un rapport des choses entre elles. Pour trouver une analogie à ce phénomène, il faut la chercher dans la région nuageuse du monde religieux. Là, les produits du cerveau humain ont l'aspect d'êtres indépendants, doués de corps particuliers, en communication avec les hommes et entre eux." (Le Capita1, Livre 1, 1867/1969:69).

L'analogie fétiche-monnaie est si tentante qu'on la retrouvera chez d'autres auteurs. Même M. Mauss, en 1934, I'appuiera de quelques arguments ethnographiques au cours d'un débat sur les fonctions sociales de la monnaie (Mauss, 1969: 116) ; un débat au cours duquel, curieusement, aucun des savants présents ne cite le nom de Marx.

De par les exigences auxquelles répond sa création, la monnaie est vouée à caractériser tout système de pure convention : un usage bien établi devient "monnaie courante". Pour souligner le caractère illusoire de telle ou telle représentation collective, Mauss et Durkheim ont parfois employé l'image de la fausse monnaie. Mauss, par exemple, comparait l'illusion à l'oeuvre dans la magie à de la fausse monnaie : "En définitive, disait-il, c'est toujours la société qui se paie elle-même de la fausse monnaie de son rêve" (1950: 119). Pour Durkheim, les formules individuelles qui tentent d'imiter l'autorité des "expressions de la pensée collective" sont "de la fausse monnaie" (1975: II-301).

Mais l'intérêt du rapprochement opéré par Marx réside dans l'analyse qui révèle la nature du produit social de l'objet-fétiche. Tant pour la monnaie et la marchandise que pour l'objet religieux, la symbolisation agit comme une perte de mémoire : l'objet inventé parâ̂t doté d'une existence et de qualités intrinsèques.

"Le yapèrè ne peut provenir du village seulement. Il faut que la brousse s'ajoute... Les racines d'un arbre ne peuvent nouer toutes seules un yapèrè. Il faut chercher beaucoup d'autres choses, les assembler, les lier. Dès que tu les as liées, et que tu as sacrifié un poulet dessus, cela te dépasse" (Oywalé Coulibali, chef de Nya, nov. 1983). 
Les rapports sociaux se sont condensés en un objet si autonome qu'on lui attribue vie, qu'on le nourrit, et qu'on lui prête des actions extraordinaires. L'objet manufacturé se présente comme un être dominateur que quelques-uns seulement peuvent tenter d'influencer. Toutefois, il n'apparaît comme une réalité indépendante qu'en faisant oublier les rapports sociaux qui le créent. Dans le langage de Marx, on dirait que le rapport humain est "mythifié" sous une forme "réifiée" (1905: IV-344). On peut à juste titre voir dans l'objet "le résultat d'une dépense du cerveau, des nerfs, des muscles des organes, des sens, etc. de I'homme, dont une fantasmagorie masque le caractère social". Indubitablement, les effets d'une forme d'activité sociale sont attribués non aux hommes mais à la chose, au produit de leur activité. Le statut d'un détenteur de yapèrè s'explique par les qualités (force, clairvoyance) d'un objet qui n'existe et ne se maintient que grâce à l'allégeance de ceux qui lui ajoutent foi. L'efficacité symbolique sans laquelle aucun système social ne peut fonctionner, s'investit ici dans une puissance (NYa, Komo, Nankon, etc.) qui revêt la forme fantastique d'une chose : le yapèrè. Reprenons librement les formulations et le raisonnement de Marx relatifs aux sociétés fondées sur la dépendance personnelle. La description concerne la société féodale et l'économie d'autosubsistance, où pour l'esentiel, la circulation des biens n'a pas de caractère marchand. Selon Marx, les rapports sociaux y apparaissent clairement comme des rapports entre les personnes. La dépendance personnelle caractérise aussi bien la production matérielle que tous les autres domaines de la vie sociale. Le travail et ses produits (qui ne passent pas par l'échange) n'ont nul besoin de prendre une forme différente de leur réalité. Ils se présentent comme une série de prestations suivies de redistributions en nature.

"De quelque manière donc, qu'on juge les masques que portent les hommes dans cette société, les rapports sociaux des personnes dans leurs travaux respectifs s'affirment nettement comme leurs propres rapports personnels, au lieu de se déguiser en rapports sociaux des choses, des produits du travail. (...) Les objets déstinés à satisfaire les besoins se présentent à la famille comme le produit du travail, et non comme des marchandises qui s'échangent réciproquement. (...) La mesure de la dépense des forces individuelles par le temps de travail apparâtt ici directement comme 
caractère social des travaux eux-mêmes, parce que les forces de travail individuelles ne fonctionnent que comme organes de la force commune de la famille". (Le Capital, I, 1867/1969:73).

Mais il reste alors à expliquer une curieuse inversion. Si "le monde religieux n'est que le reflet du monde réel" (id. p.74), comment se fait-il que des sociétés économiquement "transparentes" mettent en oeuvre des religions qui se caractérisent par l'opacité? Au point qu'une des modalités de cette opacité, le "fétichisme", serve de métaphore pour caractériser les représentations de l'économie capitaliste ! Quel est alors le statut des objets-fétiches des sociétés dont les rapports de production seraient "transparents" ?

\section{Transparence et opacité}

Observons d'abord que, depuis l'époque de Marx, les enquêtes sur le terrain se sont multipliées et ont affiné leurs méthodes. Les rapports de production à l'oeuvre dans des sociétés non capitalistes n'apparaissent plus aujourd'hui aussi transparents que le croyait Marx. Dans bien des sociétés étudiées par les anthropologues, ce sont les rapports de parenté qui règlent l'appropriation de la nature. Mais ils mobilisent des représentations qui n'ont rien d'immédiat et passent par une construction symbolique. Chez les Minyanka, par exemple, ils ne sauraient se comprendre indépendamment d'une conception "métaphysique" de la masculinité (justifiant la patrilinéarité, la patrilocalité, le pouvoir des hommes sur les femmes) et de la séniorité (justifiant la préséance des ancêtres sur les aînés et des aînés sur les cadets). D'autre part, les rapports de production ne sont pas les seuls à pouvoir se condenser en "fétiches". La puissance reconnue aux yapèrè mérite d'être analysée comme un "fait social total" car elle résulte de déterminations à la fois économiques, politiques et religieuses.

Les yapèrè sont bien plus que des produits, des utilités, ou des biens car, contrairement aux vues du Président De Brosses, au-delà de l'objet, l'existence d'une puissance est postulée. Les qualités qu'on attribue aux objets sont bien différentes de celles de leurs 
composants physiques. Aux yeux du croyant, la créature dépasse son créateur, la chose fabriquée pour servir son propriétaire devient quasiment son maitre. Ou plutôt: la chose ne sert son propriétaire que si, en retour, il la soigne et lui obéit. A défaut, elle peut se transformer en véritable fléau. Voyons quels sont les rapports sociaux qui se cachent en ces boules noirâtres supposées puissantes. Pour quelles raisons l'effort humain investi disparaît-il dans des produits que l'imaginaire projette dans le domaine religieux?

L'analogie entre le fétiche économique (monnaie ou marchandise) et l'objet religieux trouve rapidement sa limite. Le yapèrè mobilise certains rapports de production et nécessite un travail, mais il n'est évidemment pas "une méthode pour échanger du travail". Il s'agit plutôt d'un mode de reproduction des rapports de dépendance qui sous-tendent l'institution socio-religieuse. Le yapèrè est en effet indifférent à toute détermination économique qui impliquerait la loi du marché. Le preneur de yapèrè n'est pas plus un acheteur que le donneur n'est un vendeur. L'objet se paie, se transmet, se vole, mais il n'est pas voué à circuler. Son autonomisation par rapport à son procès de fabrication ne résulte pas de l'échange mais de la dépendance, de l'allégeance. Les enjeux se situant sur le plan idéologique, les notions de capital, de capitalisation, de valeur, de plus-value doivent être évitées ou employées au sens de Bourdieu : capital symbolique, plus-value symbolique, etc.

En tant que résultat d'une dépense de force vitale humaine, les yapèrè des grands cultes collectifs représentent cependant du travail matérialisé, même si notre catégorie "travail" n'existe pas comme telle dans la culture minyanka. Ils cristallisent des centaines d'heures d'efforts conjugués, et s'obtiennent contre le paiement d'une "richesse" (nafolo). Il faut d'ailleurs des années pour les confectionner et leurs parties constitutives, comme les savoirs qui s'y rapportent, se paient. Dans ce contexte, les calculs occidentaux sont bien artificiels mais ils donnent un ordre de grandeur : l'ensemble des prestations indispensables à l'installation d'un sanctuaire de Nya représente plus de 15000 FF. Aussi l'acquisition des objets de culte est-elle impossible sans le travail de tout un segment de lignage localisé. Grâce au concours de ses dépendants, 
celui qui prend l'initiative de la fondation du sanctuaire "paie" les yapèrè et devient chef de culte. L'allégeance d'autres familles, alliées au sein de la société d'initiation qui se forme, est également nécessaire; c'est parmi elles que se recrutent les autres officiants du culte : chanteurs, bouffons rituels, sacrificateurs, et le cas échéant, possédés. La fonction de chef de culte s'hérite par ordre d'âge au sein de sa descendance en ligne paternelle.

Pour le culte de Nya, du Serkolo ou du Komo, l'acquisition des yapèrè est symboliquement assimilée à un mariage. Si ce qu'on appellerait en occident la propriété d'un autel s'exprime par un mariage, c'est évidemment que le terme propriété est inadéquat. L'union du chef de culte et de la puissance amorce, comme le mariage, un pouvoir générateur de nature cosmique. Recevoir une femme et recevoir un yapèrè engendrent tous deux une dette de vie dont on ne s'acquitte jamais. Le yapèrè, comme la femme, est "acquis" par un groupe de parenté, afin d'assurer sa perpétuation. Souvent, les prestations librement consenties sont comparées au travail servile, non en raison de la contrainte qu'elles impliquent mais parce qu'elles ne se mesurent pas. En effet, de leur vivant tout au moins, les devoirs des receveurs de yapèrè envers les donneurs ne connaissent pas de terme. Aussi les seconds sont-ils souvent choisis dans un village assez lointain pour que les obligations ne soit pas trop pesantes.

Le chef d'un sanctuaire manipule une certaine richesse ; son vestibule est le point de passage d'une circulation impressionnante de dons et de contre-dons. S'il reçoit beaucoup, sous forme de bêtes sacrificielles, de bière, de pagnes et d'argent, son rôle l'oblige à redistribuer largement. Les chefs de culte vénaux, qui "travaillent comme des charlatans", sont très mal vus et leur gloire est de courte durée. La centralisation éphémère des cadeaux revêt un caractère plus socio-politique qu'économique. Par les dons et contre-dons, les individus s'obligent mutuellement, s'imposent les uns les autres des devoirs.

Le rite sacrificiel débouche, comme l'avait vu Durkheim, sur un banquet auquel les fidèles participent en même temps que les 
dieux (Durkheim, 1907/1975: II-119). Les animaux sont symboliquement adressés aux puissances religieuses, mais leurs autels n'absorbent que le sang; la viande est partagée au cours de festins bien humains, selon un ordre correspondant aux hiérarchies sociales et aux réseaux d'obligations existant entre les individus et les groupes. En somme on pourrait dire, dans le langage de Marx, qu'à l'occasion de ces distributions, l'échange d'hommes à hommes s'obscurcit en transitant par un destinataire métaphysique. Mais à y regarder de plus près, la consommation carnée qui clôture le rite manifeste son caractère social : l'ampleur des sacrifices est un bon instrument de mesure du pouvoir des chefs de culte et de la force de leurs autels. A la fin du banquet et après la redistribution des cadeaux, l'épaisseur du sang séché qui recouvre les autels constitue un résultat tangible apprécié. Cette accumulation à caractère purement symbolique révèle bien la nature du yapèrè : il demeure après le rite comme le produit coagulé des relations de pouvoir.

Sur le plan cosmique, le yapèrè se présente comme la condition de l'échange de substances entre la nature et l'homme, mais sur le plan social il condense des rapports de force complexes. Seuls certains individus sont reconnus comme opérateurs légitimes de l'échange. De ce point de vue, le yapèrè ne masque pas un rapport social, il contribue à l'établir en tant que tel et en tant que rapport au monde6. Par "réification de rapports sociaux", il faut comprendre qu'un objet joue le rôle d'opérateur logique de la constitution de ces rapports. L'objet ne vient pas en un second temps remplir une fonction de masque pour un rapport social qui lui préexisterait. Ce qui est occulté par l'objet, miraculeux, c'est la nature sociale des relations qui lui donnent sens.

La relation avec le monde référentiel (la nature, l'au-delà, les forces cosmiques) établie par un an̂né éminent grâce aux prestations de ses dépendants, se présente pour toute la communauté comme un service dispensateur de bienfaits. La croyance en l'efficacité des rites effectués par le "prédécesseur" (nyah-fo) est une des sources de la dépendance des "suiveurs" (kantugho-shyin). En outre,

\footnotetext{
6Voir les analyses de M. Augé, 1975.
} 
chacun pense devoir sa propre existence à une puissance entretenue par le travail des générations précédentes, et chacun manifeste par des sacrifices la reconnaissance de cette dette de vie.

La pratique rituelle fixe une hiérarchie dont les différents degrés sont séparés par un jeu d'interdits. I'importance statutaire des adeptes se mesure par le temps passé dans la fréquentation du sanctuaire. Elle se marque aussi par des sacrifices promotionnels qui rapprochent les fidèles de la puissance. Un premier sacrifice permet d'entrer dans la société religieuse, un deuxième autorise l'implantation d'une poterie personnelle qui contient une eau miraculeuse. Ce liquide, constitué par la décoction de racines identiques à celles qui entrent dans la fabrication des yapèrè, est absorbé sous forme de boisson ou utilisé comme lotion corporelle. L'intimité de l'initié et de la puissance est encore assurée par la consommation de la viande sacrificielle et par le port d'un petit objet personnel distinct des yapèrè collectifs mais qui reçoit sa part de sang sacrificiel. Toute succession à un office quelconque doit être marquée par le sacrifice d'un quadrupède et des poulets "de sa tête".

Dans la société minyanka, la vie politique consiste en un jeu d'équilibre entre différents pôles : chefs de famille, chef de terre, chef de village, chefs de sociétés d'initiation, associations villageoises, individus particuliers. En l'absence de foyer central de souveraineté, de loi univoque, d'institutions politiques spécialisées, c'est le sol mouvant des rapports de force qui induit des états de pouvoir7. Dans certains villages, les responsables du sanctuaire de Nya ou du Komo ont plus de poids que le chef de village.

Le recours aux yapèrè, leur mode de transmission, leur contrôle et leur gestion sont chargés de deux altérités fondamentales : 1a différence sexuelle et la différence d'âge. S'y ajoute une troisième, dépourvue de référence biologique : le travail personnel et la recherche de connaissances occultes. Les yapèrè sont "des choses d'hommes" même si certaines femmes jouent un rôle rituel marginal

\footnotetext{
${ }^{7}$ Pour une description du système politique, voir Colleyn \& Jonckers, 1983.
} 
d'hôtesse ou de ménagère d'un sanctuaire. Une femme, dit-on, ne peut s'y connaître que par le biais de la sorcellerie. Il est spécialement interdit aux femmes de toucher les instruments de culte sans y avoir été invitées, de les évoquer oralement, de pratiquer un sacrifice, bref, de se mêler des questions de yapèrè. Si la légitimité de ceux-ci est reconnue par les deux sexes, ils se présentent comme des armes spirituelles qui complètent l'arsenal idéologique masculin. Réservé aux hommes, le traitement des yapèrè vise pour une grande part à contrecarrer la sorcellerie (sinkama). Or cette dernière, qui fait planer une grave menace pour la vie sociale, apparaît comme le domaine privilégié des femmes. En matière de sorcellerie, elles ont toujours un temps d'avance sur les hommes. Selon l'expression consacrée, un homme qui divulgue les secrets liés aux yapèrè est "quelqu'un qui se donne aux femmes" (c'est-à-dire aux sorcières).

L'accession au statut d'homme, puis de chef d'une famille prospère et nombreuse, implique que 1 'on compense par la connaissance d'autels (yapèrè) le retard pris sur les femmes on matière de savoirs cachés. La démarche prend une forme défensive qui justifie aux yeux des hommes le monopole qu'ils exercent dans le domaine rituel.

La croyance en des sociétés de sorcières invisibles volant l'âme des enfants pour les incorporer dans des victimes animales, les sacrifier et les consommer, débouche sur des manoeuvres visant à intimider cet ennemi intérieur clandestin. En effet, le champ d'action privilégié de la sorcière est la communauté lignagère où elle est venue se marier. Les hommes, et surtout les aînés, sont beaucoup moins souvent accusés d'être responsables de la mort de leurs enfants. Leur force et leur savoir se mesurent par la prospérité de leur groupe de dépendance patrilinéaire. Il y a des hommes sorciers, mais d'une manière générale, "si les hommes ont des yapèrè, c'est parce que les femmes ont la sorcellerie".

En aucun cas, un cadet ne peut se procurer un yapèrè ni "faire un travail sur un yapèrè" sans en référer à ses aînés, qu'il jouisse par rapport à eux d'une autonomie économique ou non. L'obtention d'un tel objet reste soumise, ne fût-ce que formellement, à l'approbation des aînés du segment de lignage localisé. Il est également 
sévèrement interdit de se livrer à l'insu de ses aînés et à des fins personnelles, à des opérations sur des yapèrè existants : aspersion de poudres, incantations, sacrifices, prières, etc. La violation de cette règle est assimilée à une action de sorcellerie (sinkama). En revanche un chef de segment de lignage ou de famille étendue, qui n'est pas soumis à l'autorité d'un aîné, s'affilie librement à une ou plusieurs institutions socio-religieuses pour renforcer son statut et celui de ses dépendants.

Pour l'entretien des yapèrè, non seulement tous les membres du groupe de parenté au sein duquel ils peuvent s'hériter sont mobilisés, mais aussi toutes les familles qui comptent des initiés parmi leurs membres. En outre, les relations rituelles dépassent le cadre du village et s'étendent parfois sur une zone géographique assez vaste. Toute société d'initiation se situe, par rapport aux sanctuaires de même origine, dans une structure hiérarchique transvillageoise liée à I'ancienneté de leurs autels. La capacité d'accumuler des yapèrè forme donc un système institutionnel qui définit les statuts sociaux et dresse l'enceinte des luttes d'influence.

L'activité religieuse prend en charge la répression des atteintes aux usages institutionnalisés et la régulation des conflits. Chaque sanctuaire - et il peut $y$ en avoir plusieurs par village - définit un espace structuré obéissant à des lois de fonctionnement. En raison de l'âge, de 1 'ancienneté, des relations de parenté, d'alliance et d'allégeance, le culte met en oeuvre un système hiérarchique. Comme ill est dirigé par un collège formé d'ainés, son activité ressemble, au premier abord, à une conspiration d'hommes âgés soucieux de renforcer leur autorité sur leurs dépendants. Lors des grandes cérémonies de Nya, du Manyan, du Nankon et du Komo, fréquentes sont les scènes où les aînés (chefs de Nya, témoins, possédés, forgerons) s'accroupissent pour chuchoter, tandis que la jeunesse semble absorbée par les chants et la danse. Mais les aînés n'apparaissent pas comme les véritables détenteurs du pouvoir : les yapèrè sont considérés comme l'instrument des ancêtres fondateurs du culte. Les sacrifices offerts à la puissance sont d'ailleurs toujours confiés à leurs soins. Les incantations comprennent des demandes 
aux ancêtres, supposés plus capables de faire agir les yapèrè. Cette mainmise des ancêtres, qui de l'au-delà continuent à contrôler les yapèrè, donne aux aînés les moyens de s'effacer. Soucieux de prouver l'innocence d'un pouvoir basé sur l'âge, ils s'infantilisent devant des puissances plus âgées encore. Les prières commencent toujours par la même formule : "Je ne suis qu'un enfant; je ne distingue pas ma main droite de ma gauche..." Cette humilité n'est pas exempte d'ostentation : il n'y aurait pas d'aînés, seulement des enfants qui interprètent les volontés des vieux, aujourd'hui disparus. La manipulation des yapèrè ne peut non plus s'interpréter seulement en termes de répression. D'abord parce que l'accord des dignitaires d'un culte n'est jamais acquis d'avance : souvent, les rivalités ou les conflits d'intérêts qui les opposent compliquent les stratégies. Il n'est pas rare qu'un cadet ou une femme voie son point de vue approuvé par le jeu d'une coalition complexe. Ensuite, parce que la mise en scène rituelle fondée sur la possession (Nya, Nankon, Sandogno, Serkolo, Duba, Dagoro) ou sur les masques (Komo, Kono, Namakoro, Tyi-wara) déploie aussi bien des procédures de réconciliation que des techniques de coercition.

Quand une famille est enlisée dans des conflits internes, l'intervention extérieure et les médiations organisées par les officiants permettent souvent de débloquer la situation. C'est certainement une des fonctions de ces institutions socio-religieuses. Le chef de famille a beaucoup de chances de voir sa position renforcée mais ses manières d'agir peuvent être mises en cause. On voit des â̂nés redouter la médiation des dignitaires responsables des sacrifices aux yapèrè. Jamais cependant, un cadet ne pourra venir se plaindre de son chef de famille aux responsables d'un sanctuaire. Ceux-ci ne prennent les conflits en compte que lorsqu'ils empoisonnent les relations sociales, et qu'il n'est plus possible de faire autrement.

Un litige entre deux personnes est toujours transformé (par un devin ou un porte-parole attitré) en deux relations individuelles avec la puissance religieuse : c'est Nya, le Komo ou le Nankon que celui qui a tort a offensé. Ce glissement de responsabilité présente un avantage évident : personne ne peut se sentir humilié de devoir rendre des comptes à une entité supra-humaine. Les biens exigés 
par celle-ci ne visent d'ailleurs jamais à réparer un dommage; ils consistent en offrandes adressées à la puissance, qui sont redistribuées par le chef de culte.

La théorie de la sorcellerie donne forme à un rapport de force au niveau familial et villageois : si un cadet ose se procurer un yapèrè, si une femme ose hausser le ton pour critiquer les hommes, c'est qu'ils s'appuient sur une connaissance liée à la sorcellerie. Or la pratique de la sorcellerie déclenche automatiquement la fureur des yapèrè. Le poids des intimidations, l'autorité du complexe puissance-yapèrè provoque souvent l'aveu de la personne incriminée ou soupçonnée; à moins qu'il s'agisse d'une manière "hérol̈que" de se libérer de pulsions agressives.

En se clôturant par une séance médiumnique au cours de laquelle le possédé ou le porteur de masque chuchote le vrai sur toutes choses, une fête de Nya, du Nankon ou du Komo actualise un rapport de force et lui donne son sens. Elle fournit également une grille d'interprétation des aléas de la vie. Quand quelqu'un tombe gravement malade par exemple, lui-même aussi bien que son entourage spéculent sur les causes du mal. Le devin consulté peut mettre en cause une puissance à yapèrè, à moins que ce ne soit le possédé ou le porteur de masque qui ne révèle l'étiologie de la maladie : rupture d'interdit, désobéissance à un aîné, ou action sorcière. Des sacrifices au yapèrè seront alors prescrits.

Ces cérémonies admettent donc une dimension quasiment judiciaire, mais les délibérés ne peuvent se comparer à ceux d'un tribunal. Les aînés, et même les cadets si on les y autorise, discutent le cas et marchandent les offrandes exigées. Le "dialogue avec les dieux" n'est pas exempt d'insultes, de moqueries, de contestations. De son côté, la rumeur publique colporte également des diagnostics qui, à l'occasion de malheurs, de maladies, de décès, font souvent intervenir les yapèrè. Tous ces jugements se présentent moins comme le constat de sentences réellement exécutées que comme des interprétations a posteriori confortant un rapport de force : "Untel s'est attaqué à plus fort que lui", "Untel a rompu les interdits de Nya", "Unetelle s'est disputée avec le chef du Komo", etc. En somme, le code fonctionne 
selon le principe : tel acte est considéré comme fautif parce que les événements vécus par celui qui l'a commis peuvent être considérés comme une conséquence méritée.

Jusqu'à la fin de l'époque coloniale, les noms des personnes "tuées par les yapèrè" étaient clamés bien haut. Dans les cas de sorcellerie, leurs habits étaient étalés sur le toit des sanctuaires. De nos jours encore, on considère que ces puissances exécutent les gens qui les ont défiées en rompant des interdits, mais on se fait très discret sur cette question. Les dignitaires du culte craignent que l'administration, qui s'occupe davantage que par le passé des affaires villageoises, ne fasse une enquête sur de tels décès. Les risques de conflits de droits se multiplient et les adeptes des cultes traditionnels en sont pleinement conscients. Nya, le Komo et d'autres puissances cessent tout doucement d'apparaître comme l'expression de la violence sociale légitime, ce qui concourt à leur progressive perte de crédit. La nature politique de l'organisation porteuse de yapèrè transperce cependant encore dans les chants: "Le Commandant 8 parle aujourd'hui de pouvoir, notre Nya en parle éternellement" (décembre 1986).

\section{Conclusion}

L'étude du statut de ces objets dans le système des représentations et des pratiques sociales fait clairement ressortir que religion, magie et sorcellerie ne peuvent être posées comme des domaines séparés. Elles s'intègrent toutes trois dans ce que les Minyanka appellent le Bamanaya, qui correspond à un mode de vie et à un système de pensée que Comte eut qualifié de fétichiste et Tylor d'animiste. Le débat sur le fétichisme s'est souvent enlisé dans les querelles de mots et il n'est pas certain que les objets "forts" de tous les peuples de la terre soient justiciables d'un même concept. De toute façon, une définition formaliste de ce qu'est un "fétiche"

8Titre désignant le Chef d'Arrondissement ou le Commandant de Cercle. 
ne présente pas grand intérêt: sans doute ne parle-t-on de la même chose qu'à l'intérieur de ce qu'il faut bien appeler une aire culturelle. Curieusement, la fascination que les objets religieux ont exercée sur les auteurs tels Comte, Tylor, Mauss, Haddon, Malinowski et bien d'autres, a oblitéré un aspect de leur réalité. Tout se passe comme si les objets avaient masqué le caractère social des relations qui les font exister et auxquels ils sont nécessaires.

Renonçons, s'il le faut, aux termes de fétiche et de fétichisme, qui ne peuvent plus prétendre à la neutralité scientifique, mais retenons quelque chose de l'idée marxiste d'une "fétichisation" des rapports sociaux. Cette idée nous aide à comprendre que dans un système politique acéphale comme celui des Minyanka, rapports sociaux et artéfacts symboliques s'impliquent réciproquement. De ce point de vue, l'anthropologie religieuse et la sociologie ne se séparent pas en disciplines distinctes. Ies différenciations sociales, dans ce cas-ci construites à partir du sexe, de l'âge, et du savoir, sont au coeur du symbolique sans lequel elles ne sauraient s'établir. Religion, magie et sorcellerie forment un système unique qui soulève la question majeure de la croyance. C'est en effet le legs des ancêtres, l'héritage d'un fonds symbolique commun qui permet aux hommes de s'entendre pour diviser le temps en cycles, pour poser leur travail rituel comme condition vitale et pour établir des rapports de dépendance interpersonnels. Si les résultats du travail rituel ne sont pas toujours aussi favorables qu'on l'escomptait, l'attente n'est jamais non plus tout à fait déçue; même pour les morts, qui ont toujours leur mot à dire dans l'au-delà, la vie continue.

La vie sociale des Minyanka ne se règle pas à coups de griffes et de dents. Nous n'avons pas affaire à des gens plus angoissés que d'autres. Comme tout le monde, ils se préoccupent des menaces qui pèsent sur eux et trouvent dans leur culture des modèles d'interprétation. Ils ne sont pas obsédés par les agressions de sorcellerie, mais ils s'en soucient et s'intéressent aux techniques d'auto-protection. Dans cette société sans grande mobilité sociale, sans concurrence économique véritable, une lutte compétitive se livre 
dans l'ombre pour l'obtention de yapèrè, de poudres, de formules secrètes. En l'absence d'autorité politique centralisée, les sanctuaires abritant les yapèrè constituent des pôles de pouvoir permettant l'expression des luttes d'influence. Les individus, hommes et femmes, acceptent les règles d'un jeu qu'ils connaissent et les manipulent. Leurs désirs et leurs craintes s'incarnent en des objets puissants. Mais la force de l'objet est toujours plus ou moins consciemment mise en relation avec l'envergure sociale de celui qui le détient comme de celui qui le brave.

On pourrait ici tirer profit de certaines analyses que Pierre Bourdieu a consacrées aux luttes symboliques. N'oublions pas que la principale qualité d'un yapèrè, c'est d'être dangereux, mauvais, méchant. C'est pourquoi il est le signe distinctif des individus classés comme forts. Mais ce jeu de la distinction n'a rien d'ostentatoire: il s'agit de parâtre, non en montrant mais en suggérant. Il faut donner l'impression d'être fort en cachant ce que l'on sait. Dans ce contexte, I'âge est une valeur politique: "avoir duré dans les affaires de yapèrèl est un exploit tenu en haute estime. Cela signifie qu'on a pu vaincre ses ennemis grâce à la manipulation d'objets souverains, sans y succomber soi-même. C'est un exercice auquel on affecte d'importantes ressources et auquel on consacre beaucoup d'énergie.

En partageant les préoccupations quotidiennes des initiés, on s'aperçoit que la connaissance secrète de la composition des yapèrè, la connivence de ceux qui prétendent savoir, la recherche des valeurs ajoutées et le serment de discrétion, procurent un immense plaisir. Comme la culture élitiste de notre société, l'accession à l'art de la bamanaya est "un enjeu social qui suppose et impose à la fois qu'on entre dans le jeu et qu'on se prenne au jeu" (Bourdieu, 1979: 279). La quête qu'on eût naguère qualifiée de "fétichiste" offre le prétexte à de multiples intrigues. Les démarches entreprises pour obtenir un savoir qui ne cesse de se dérober et la rouerie nécessaire pour égarer les curieux sont l'ornement de la vie de ceux qui sacrifient aux yapèrè. En outre, les grandes cérémonies semestrielles destinées à "nourrir" les yapèrè baignent dans une atmosphère festive, avec abondance de viande, de bière et de discus- 
sions sur les grands thèmes de la vie aussi bien que sur les rumeurs qui agitent l'opinion.

Une des fonctions essentielles des yapèrè publics est de fournir des procédures d'arbitrage aux conflits qui débordent le cadre domestique. Seul un pouvoir supra-humain peut obtenir d'un individu qui se respecte, des excuses et des offrandes de réparation. Les cultes actualisent périodiquement l'allégeance des adeptes par le travail rituel qui leur est demandé. Ils fournissent aussi une explication aux adversités en même temps qu'ils proposent le moyen de les infléchir. Ces interprétations sont toujours travaillées par les tensions inévitables dans un milieu social of I'on vit près les uns des autres. Dans la chronique d'un tel milieu, c'est toujours l'incident qui fait l'événement. Le cours heureux des choses appelle peu de commentaires.

\author{
Jean-Paul Colleyn \\ Laboratoire Associé $n^{\circ} 221$ \\ CNRS - EPHE
}

\title{
Bibliographie générale
}

Adler A.

1970 "L'ethnologie et les fétiches" in objets du fétichisme, Augé $M$.

Nouvelle Revue de Psychanalyse, 2, Paris.

1975 Théorie des pouvoirs et idéologie, Paris, Hermann.

Baudrillard J.

1970 "Fétichisme et idéologie: la réduction sémiologique" in Objets du fétichisme, Nouvelle Revue de Psychanalyse 2, Paris.

Bonnafé P.

1970 "Objet magique, sorcellerie et fétichisme?" in Objets du fétichisme, Nouvelle Revue de Psychanalyse 2, Paris.

Brosses (de)

$1760 \mathrm{Du}$ culte des dieux fétiches, ou parallèle de l'ancienne religion de l'Egypte avec la religion actuelle de Nigritie.

Bourdieu $P$.

1979 La distinction. Critique sociale du jugement. Paris, Ed. de Minuit. 
Comte A.

1908 Cours de Philosophie Positive, Paris, Schleicher.

Durkheim E.

1913 Les formes élémentaires de la vie religieuse, rééd. PUF 1979.

1975 Textes T.II, Religion, morale, anomie, Paris, Ed. de Minuit.

Griaule $M$.

1940 "Remarques sur le mécanisme du sacrifice dogon", Journal de la Société des Africanistes X, 27-130.

Haddon A.

1906 Magic and fetishism, London, Free Press.

Hegel G.W.F.

1830 La raison dans 1'histoire, rééd., UGE, 10-18, 1965.

Hervé $P$.

1955 La Révolution et les fétiches.

Lévi-Strauss C.

1950 "Introduction à l'oeuvre de Mauss", in M. Mauss, Sociologie et anthropologie, Paris, PUF.

1962 La Pensée Sauvage, Paris, Plon.

Marx $\mathrm{K}$.

1859 Contribution à la critique de l'économie politique. Rééd. Editions Sociales, 1977.

1867 Le Capital, Livre I, rééd. Garnier-Flammarion, Paris, 1969.

1905 Le Capital, Livre IV. Théories sur la plus-value,. T.III, Paris, Editions Sociales, 1976.

Mauss $M$.

1950 Sociologie et anthropologie, Paris, PUF.

1968 0euvres. T.I, Les fonctions sociales du sacré, et

1969 Deuvres, T.II, Représentations collectives et diversité des civilisations, Paris, Ed. de Minuit.

Pontalis J.B.

1970 "Présentation" in Objets du f'étichisme, Nouvelle Revue de Psychanalyse, 2, Paris.

Pouilion J.

1970 "Fétiches sans fétichisme", in Objets du fétichisme, Nouvelle Revue de Psychanalyse, 2, Paris.

Sahlins M.

1980 Au coeur des sociétés, Paris, Gallimard.

Tylor E.B.

1871 Primitive Culture (Ed. franc. La Givilisation Primitive, 1876). 


\section{Références sur les Minyanka}

Bertaux C. et Jespers Ph.

1981 "Quelques opérations sacrificielles liées aux géomancies bambara et minyanka du Mali", Systèmes de Pensée en Afrique Noire, 5, 71-98.

Colleyn J.P.

1975 "Notes sur la pensée religieuse des Minyanka du Mali", Systèmes de Pensée en Afrique Noire, vol.1, 19-34.

1982 'Le chiot court mais ne connât pas les odeurs': notes sur la société et les systèmes de pensée des Minyanka du Mali", Africa, LII, 1, 3-14.

Jespers Ph.

1976 "Contribution à l'étude des autels sacrificiels du Nya chez les Minyanka du Mali", Systèmes de Pensée en Afrique Noire, $2,111-139$.

1979 "Signes graphiques minyanka", Journal de la Société des Africanistes, XLIX-1, 71-102.

1983 "L'arc et le sang des chiens", Systèmes de Pensée en Afrique Noire, 6, 65-102.

Jonckers

1976 "Contribution à l'étude du sacrifice chez les Minyanka", Systèmes de Pensée en Afrique Noire, 2, 91-110.

1981 L'organisation socio-économique des Minyanka du Mali, Univ. Libre de Bruxelles, Inst.d'Ethnologie du Musée de l'Homme, série 1982, microfiche 820324.

1986 "Les 'faiseurs d'enfant', réflexions sur le statut des femmes dans le système religieux minyanka", Journal de la Société des Africanistes, LVI-1 (sous presse).

1986 "Hiérogamie et stratégies matrimoniales" in F. Héritier (éd.), La Complexité de l'Alliance, I, Archives Contemporaines, coll. Ordres Sociaux (sous presse).

Jonckers D. et Colleyn J.P.

1983 "Ceux qui refusent le mâtre : la conception du pouvoir chez les Minyanka du Mali", Africa, LIII-4, 43-58. 